\title{
Temperature Dependence of Magnetophotoconductance in One- Dimensional Molecular Assembly of Hexabenzocoronene
}

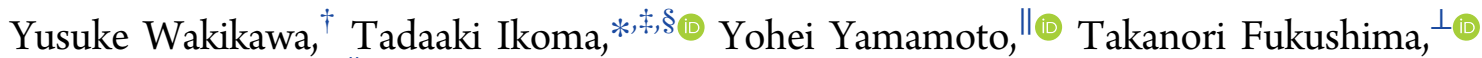
and Kimio Akiyama

${ }^{\dagger}$ Advanced Instrumental Analysis Center, Shizuoka Institute of Science and Technology, 2200-2 Toyosawa, Fukuroi, Shizuoka
$437-8555$, Japan
${ }^{\ddagger}$ Institute for Science and Technology and ${ }^{\S}$ Center for Coordination of Research Facilities, Niigata University, 2-8050 Ikarashi,
Nishi-ku, Niigata 950-2181, Japan
${ }$ Faculty of Pure and Applied Sciences, University of Tsukuba, 1-1-1 Tennodai, Tsukuba, Ibaraki 305-8573, Japan
${ }^{\perp}$ Laboratory for Chemistry and Life Science, Institute of Innovative Research, Tokyo Institute of Technology, 4259 Nagatsuta,
Midori-ku, Yokohama 226-8503, Japan
${ }^{\#}$ Institute of Multidisciplinary Research for Advanced Materials, Tohoku University, 2-1-1 Katahira, Aoba-ku, Sendai 980-8577, Japan

Supporting Information

ABSTRACT: Temperature dependencies of transient photocharge and magnetophotoconductance effect of columnar self-assemblies of the hexabenzocoronene derivative (HBC-C14), which is a prospective onedimensional photoconductor, presented different thermal activation processes for carrier generation and transportation, respectively. Thermal equilibrium between the low-lying short distance and high-lying longdistance geminate electron-hole $(\mathrm{e}-\mathrm{h})$ pairs is the origin for activation in carrier generation. The energy difference between these $\mathrm{e}-\mathrm{h}$ pairs is estimated to be $7 \mathrm{meV}$, which was mainly due to the Coulomb interaction. On the other hand, the carrier transport with thermal activation was understood by the multiple trapping model. Carrier detrapping from localized states located in the band gap causes the thermal activation in the carrier transport. The shallow energy depth at the density peak of the localized state from the mobility edge $(10 \pm 3 \mathrm{meV})$ is a unique nature of

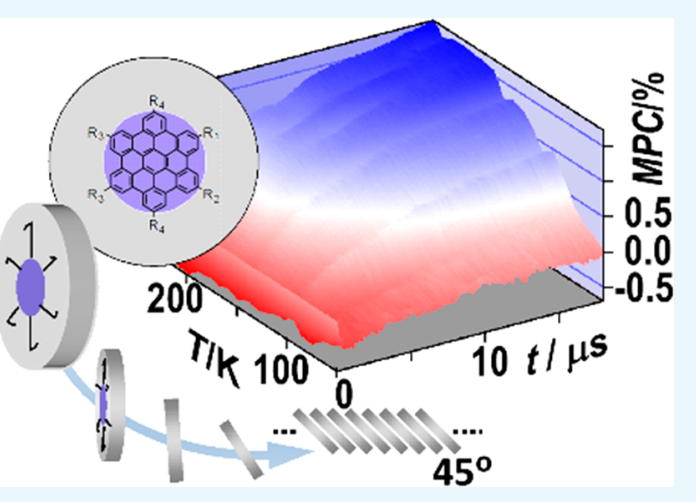
HBC-C14 self-assemblies. A very narrow Gaussian distribution for density of the localized states was also clarified.

\section{INTRODUCTION}

Photoconduction in organic semiconductors, which is an important physical property for organic photovoltaics and photosensors, can be interpreted in terms of two processes, which are primary carrier photogeneration and following carrier transport. There are two steps in carrier photogeneration. ${ }^{1}$ Initially, a Coulombically bound electron-hole $(\mathrm{e}-\mathrm{h})$ pair is generated from an exciton injected by photon absorption. Subsequently, the photogenerated geminate $\mathrm{e}-\mathrm{h}$ pair undergoes either charge recombination or dissociation, generating free charged carrier on a potential formed by the binding Coulomb field and an external electric field $(E)$. The carrier transport is basically the diffusion and drift motions of the free carrier under the effective $E$ field. ${ }^{2}$ In this carrier transport, the free carrier possibly encounters another carrier, a trap, an impurity, or an exciton and then forms various nongeminate pairs, which can act as important precursor for annihilation of the free carrier. Hence, pair dynamics is a key elementary process in both carrier generation and transport. The measurement of the magnetophotoconductance (MPC) effect originated from the interplay between the spin conservative recombination and intersystem crossing (isc) of the pair, as illustrated in Figure 1a, allows to study the $\mathrm{e}-\mathrm{h}$ pair dynamics because the isc is influenced by the external magnetic field (B). ${ }^{3-5}$

Hexabenzocoronene attached with six tetradecyl chains (HBC-C14, see Figure 1b) organizes highly ordered columnar assembly because of the strong $\pi-\pi$ interactions between the aromatic cores. $^{6-10}$ Because of this unique nanostructure, HBC-C14 shows one-dimensional photoconductivity, with high hole mobility. ${ }^{11}$ To effectively use the promising HBC-C14 for electronic devices, ${ }^{12}$ it is required to clarify further the mechanism of photoconduction in the one-dimensional molecular assembly. In this article, therefore, we studied photoconduction of the HBC-C14 assembly by means of timeresolved measurement of the MPC effect, which enables the separation of carrier generation and transport in the time domain. We will show thermal activation processes in the $\mathrm{e}-\mathrm{h}$

Received: April 19, 2017

Accepted: June 20, 2017

Published: July 7, 2017 


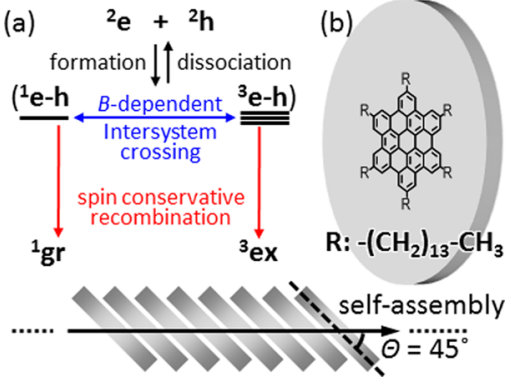

Figure 1. (a) Elementary kinetics in the $\mathrm{e}-\mathrm{h}$ pair mechanism for the MPC effect in an organic semiconductor and (b) structure of a onedimensional assembly consisting of stacked HBC-C14 disks.

pair dynamics in both carrier generation and transport on the basis of temperature dependence of the MPC effect in the early and later delay times. Kinetic analysis of the observed temperature dependencies presents an equilibrium between the short and long-distance $\mathrm{e}-\mathrm{h}$ pairs in carrier generation and a narrow distribution for density of the shallow localized states in carrier transport.

\section{RESULTS}

Figure 2a shows a typical transient photocharge $(Q)$ signal for HBC-C14 columnar objects observed under excitation by a

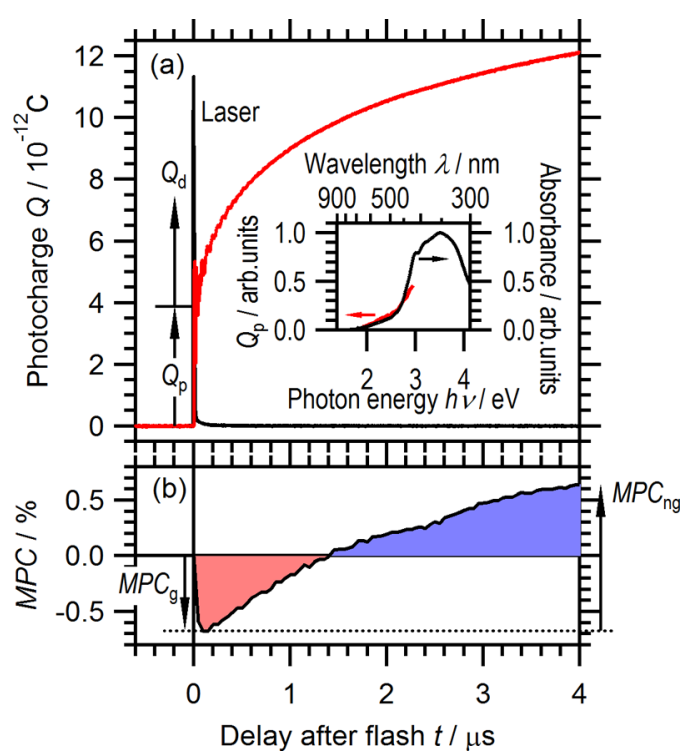

Figure 2. Time profiles of the photocharge (a) and the MPC effect at $B=50 \mathrm{mT}$ (b) for HBC-C14 self-assembly film observed by photoexcitation at $\lambda=355 \mathrm{~nm}$ under $T=200 \mathrm{~K}$ and $E=2.0 \times 10^{5} \mathrm{~V} /$ $\mathrm{cm}$. Inset of a is optical absorption (black) and $Q_{\mathrm{p}}$-detected excitation spectra (red) of the HBC-C14 self-assembly measured at $298 \mathrm{~K}$.

laser, with a wavelength $(\lambda)$ of $355 \mathrm{~nm}$, at a temperature $(T)$ of $200 \mathrm{~K}$. The $Q$ signal is detected immediately after the laser pulse and then increased slowly with the delay time after laser flash $(t)$. The prompt signal of the photocharge $\left(Q_{p}\right)$ is proportional to the initial quantity of the photocarrier born from the geminate $\mathrm{e}-\mathrm{h}$ pair. The delayed component after the flash $\left(Q_{d}\right)$ arises from drift motion of the free carrier under an $E$ field, corresponding to the carrier transport. The saturation behavior of $Q_{d}$ with the delay time reflects elimination of the free carriers, such as trapping, nongeminate recombination, and so on. The action spectrum of $Q_{p}$ agrees with the optical absorption spectrum, of which the peaks at $2.96 \mathrm{eV}$ are characteristic of the HBC-C14 columnar assembly. ${ }^{13,14}$ This fact indicates carrier generation from the singlet excitons in the HBC-C14 column. Figure $2 \mathrm{~b}$ depicts transient MPC of the HBC-C14 column detected at $B=50 \mathrm{mT}$. The observed MPC effect, which is defined by $\operatorname{MPC}(B, t)=[Q(B, t)-Q(0, t)] \times$ $100 / Q(0, t)$ as a function of $B$ and $t$, appeared in a negative phase in the early times. After the time when MPC reaches a negative maximum, the effect grows up in a positive direction and changes its phase from negative to positive at $t=1.4 \mu \mathrm{s}$. The negative fast component is caused by the geminate singlet $\mathrm{e}-\mathrm{h}\left({ }^{1} \mathrm{e}-\mathrm{h}\right)$ pair born from the singlet exciton $\left({ }^{1} \mathrm{ex}\right){ }^{3,15}$ The isc from ${ }^{1} \mathrm{e}-\mathrm{h}$ to the triplet $\mathrm{e}-\mathrm{h}\left({ }^{3} \mathrm{e}-\mathrm{h}\right)$ pair occurs quickly in the absence of $B$, as illustrated in Figure 1a. Although the ${ }^{1,3} \mathrm{e}-\mathrm{h}$ pairs of $\mathrm{HBC}-\mathrm{C} 14$ respectively recombine to the ground singlet state $\left({ }^{1} \mathrm{gr}\right)$ and the triplet exciton $\left({ }^{3} \mathrm{ex}\right)$ according to the spin conservation rule, the rate for singlet recombination is faster than that for the triplet one. On the other hand, because the Zeeman interaction that partially solves the degeneracy among the spin sublevels of the $\mathrm{e}-\mathrm{h}$ pair suppresses the isc rate, the recombination efficiency of the $\mathrm{e}-\mathrm{h}$ pair increases in the presence of $B$, resulting in the negative MPC effect due to decrease of charge-carrier density. The origin of the positive slow component is assigned to the nongeminate $\mathrm{e}-\mathrm{h}$ pair because the nongeminate pair can be formed even at later times and the population of the ${ }^{3} \mathrm{e}-\mathrm{h}$ pair is statistically higher than that of ${ }^{1} \mathrm{e}-\mathrm{h}$ pair. The positive phase arises from the carrier density enhanced by dissociation from the highest and lowest Zeeman spin sublevels of the ${ }^{3} \mathrm{e}-\mathrm{h}$ pair under the external $B$ field.

Figure 3a shows temperature dependence of the photocharge observed in excitation at $\lambda=355 \mathrm{~nm}$. With decrease in $T$, the $Q_{p}$ intensity decreases and the growth speed of $Q_{d}$ becomes slow, indicating, respectively, decrease of the carrier photogeneration efficiency and deceleration of the drift motion in low temperatures. In a semilog plot of the photocharge versus the reciprocal of temperature, the so-called Arrhenius plot of the photocharge (Figure $3 b$ ) shows a linear relationship, in which the slope changes by 1 order of magnitude at a critical temperature $\left(T_{c}\right)$ of ca. $90 \mathrm{~K}$. Therefore, the temperature region can be divided into high and low $T$ regimes at $T_{\mathcal{c}}$ in which the apparent thermal activation energies are, respectively, estimated to be $\Delta E^{\mathrm{HT}} \sim 15 \mathrm{meV}$ and $\Delta E^{\mathrm{LT}} \sim 1.5 \mathrm{meV}$. The slope in the low $T$ regime mostly remains constant at any delay time, whereas that in the high $T$ regime varies in the time region when the free carrier transports along the $E$ field. The different time behaviors of the slope indicate that the activation energy in the low $T$ regime is relevant to a thermal process in carrier generation and that in the high $T$ regime comes from another thermal activation in the carrier transport.

We measured temperature dependence of the MPC effect at $B=50 \mathrm{mT}$, which is much larger than that of the hyperfine interactions causing the coherent isc between the ${ }^{1,3} \mathrm{e}-\mathrm{h}$ pairs (see Figure S1) in organic semiconductor. As shown in Figure $4 \mathrm{a}$, the prompt negative MPC due to the geminate $\mathrm{e}-\mathrm{h}$ pair just after laser excitation appears in both the high and low $T$ regimes, whereas the obvious positive MPC due to the nongeminate pair at later times is detected only in the high $T$ regime. Temperature dependencies of the MPCs at early $(t=$ $200 \mathrm{~ns})$ and late $(t=3 \mu \mathrm{s})$ times are depicted in Figure 4b. The negative MPC at $200 \mathrm{~ns}$, which is almost a purely negative effect of the geminate pair mechanism, decreases with 

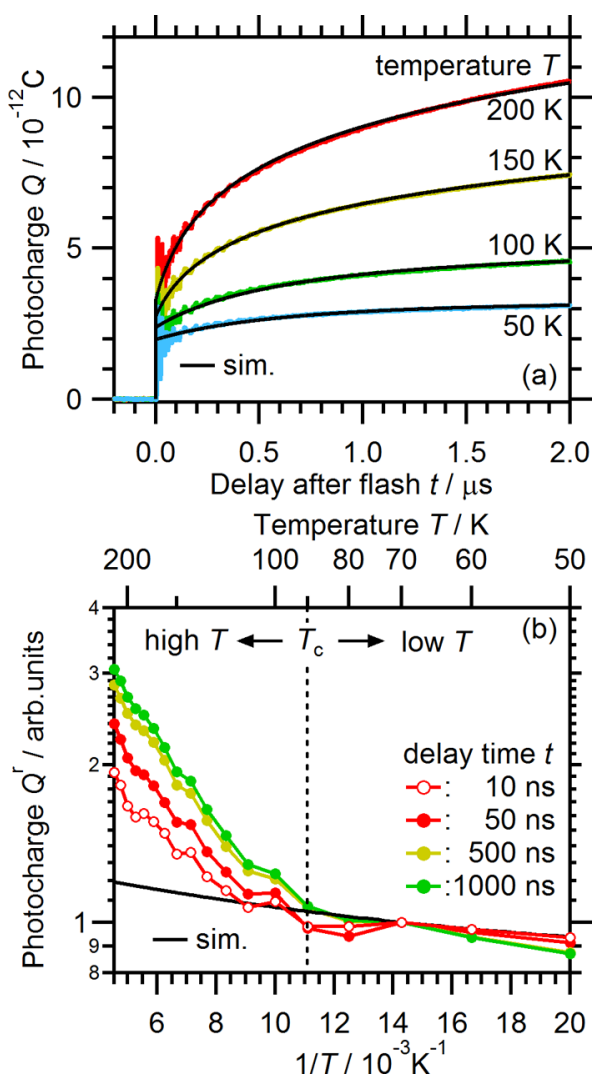

Figure 3. (a) Temperature dependence of the photocharge and (b) inverse temperature dependence of the logarithm of the photocharge observed by photoexcitation at $\lambda=355 \mathrm{~nm}$ under $E=2.0 \times 10^{5} \mathrm{~V} / \mathrm{cm}$. The plots in $\mathrm{b}$ were normalized at $T=70 \mathrm{~K}$ for ease of comparison of the slopes. The black curves in $\mathrm{a}$ and $\mathrm{b}$ are simulations calculated by the kinetic analysis considering the thermal activation processes for carrier transport and generation, respectively (see text).

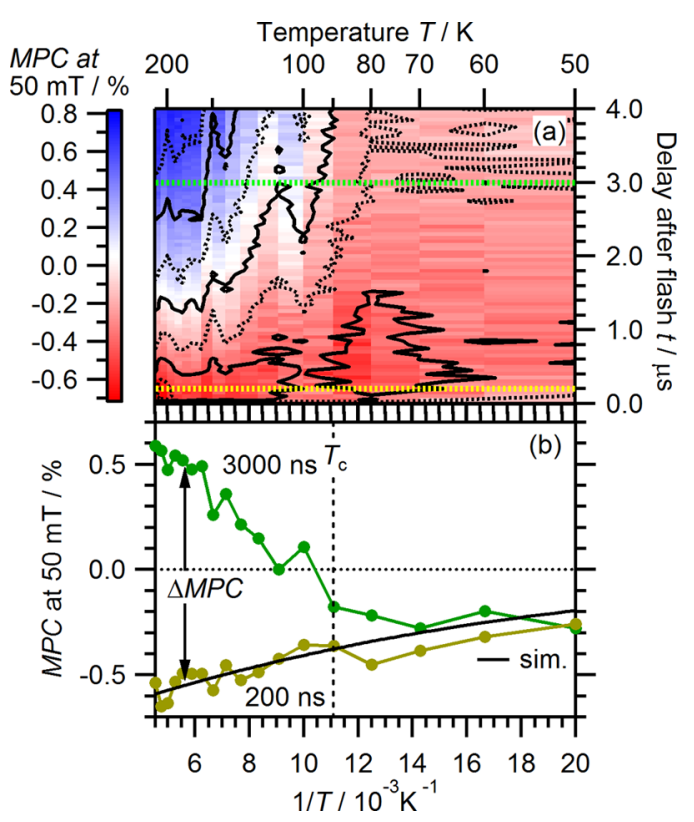

Figure 4. (a) Temperature dependence on the time profiles of MPC observed at $B=50 \mathrm{mT}$ under $E=2.0 \times 10^{5} \mathrm{~V} / \mathrm{cm}$. (b) Plots of the MPC sliced at $t=200 \mathrm{~ns}$ (yellow) and $3 \mu \mathrm{s}$ (green). The black line is the simulation curves based on carrier generation, including the thermal process with activation energy of $7 \mathrm{meV}$ (see text). decreasing $T$. The subtraction of MPCs at $200 \mathrm{~ns}$ from those at $3 \mu \mathrm{s}(\triangle \mathrm{MPC})$, which reflects a positive effect only for the nongeminate pair mechanism, also decreases by lowering $T$. The observed temperature dependencies of the geminate and nongeminate MPC effects, respectively, indicate thermal activation processes in carrier generation and transport.

The relative dissociation efficiency of the geminate $\mathrm{e}-\mathrm{h}$ pair $\left(\phi_{\mathrm{d}}{ }^{\mathrm{r}}\right)$ that was obtained by $Q_{\mathrm{p}} / E$ drastically increased with increasing $E$ (see Figure S2), which was interpreted in terms of deformation of the Coulomb potential between e and $h$ of the geminate pair by the applied external $E$ field. Slope of the observed electric field effect decreased with decreasing $T$. The Onsager theory, considering the potential deformation by the $E$ field, ${ }^{16-18}$ simulates the electric field effect observed at room temperature, but the temperature dependence predicted by the same theory does not agree with the observed one. The deviation from the theory, in which an infinite recombination is taken into account, strongly implies a temperature-dependent recombination rate of the geminate $\mathrm{e}-\mathrm{h}$ pair in carrier generation. $^{19-21}$

\section{DISCUSSION}

3.1. Carrier Generation. The $Q_{P}$ intensity of the $\mathrm{HBC}-\mathrm{C} 14$ assembly slightly increased with increasing $T$ in the low $T$ regime, manifesting a small activation energy for dissociation from the geminate $\mathrm{e}-\mathrm{h}$ pair in the carrier generation, which is not interpreted by the Onsager theory. To the best of our knowledge, a hopping dissociation explains the activated behavior in a random organic system, ${ }^{22}$ but there is no good model for the organized system. The MPC, due to geminate recombination in the $\mathrm{HBC}-\mathrm{C} 14$ assembly, negatively increased with increasing $T$, indicating that the same activation is also relevant to the $B$ field-dependent isc of the geminate $\mathrm{e}-\mathrm{h}$ pair. To understand the observed thermal activation of both dissociation and isc, we discuss about the geminate pair dynamics, with a model including two types of $\mathrm{e}-\mathrm{h}$ pairs: one is a short distance pair $\left(e-h_{r}\right)$ that is a precursor for the recombination, with a rate constant of $k_{\mathrm{r}}$, and another is a longdistance pair $\left(\mathrm{e}-\mathrm{h}_{\mathrm{d}}\right)$ from which the dissociation takes place, with a rate constant of $k_{\mathrm{d}}$, as illustrated in Figure 5. The $\mathrm{e}-\mathrm{h}_{\mathrm{d}}$ is assumed to be located on top of the geminate pair potential, which is formed in a balance between the Coulomb interaction and external $E$ field. The energy gap between these pairs $\left(\Delta E_{\mathrm{g}}\right)$ is the origin for activation energy in carrier generation. Separation from $\mathrm{e}-\mathrm{h}_{\mathrm{r}}$ to $\mathrm{e}-\mathrm{h}_{\mathrm{d}}\left(k_{\mathrm{up}}\right)$ requires extra energy of $\Delta E_{\mathrm{g}}$, but approach $\left(k_{\mathrm{dn}}\right)$ and dissociation $\left(k_{\mathrm{d}}\right)$ of the $\mathrm{e}-\mathrm{h}_{\mathrm{d}}$ need no activation energy. Not only the Coulomb interaction but also the exchange interaction $(J)$, which rapidly increases with decreasing separation distance $(r)$, seriously influences the potential of short distance pair. Hence, a large $J$ value of the e$\mathrm{h}_{\mathrm{r}}$ splits the potential into the singlet $(\mathrm{S})$ and triplet $(\mathrm{T})$ states.

Rate equations of the geminate $\mathrm{e}-\mathrm{h}$ pairs are written as

$$
\begin{aligned}
& \left\{\begin{array}{l}
\frac{\mathrm{d} n_{\mathrm{e}-\mathrm{h}_{\mathrm{r}}}}{\mathrm{d} t}=-\left(k_{\mathrm{r}}+k_{\mathrm{up}}\right) n_{\mathrm{e}-\mathrm{h}_{\mathrm{r}}}+k_{\mathrm{dn}} n_{e-\mathrm{h}_{\mathrm{d}}} \\
\frac{\mathrm{d} n_{\mathrm{e}-\mathrm{h}_{\mathrm{d}}}}{\mathrm{d} t}=k_{\text {up }} n_{\mathrm{e}-\mathrm{h}_{\mathrm{r}}}-\left(k_{\mathrm{dn}}+k_{\mathrm{d}}\right) n_{\mathrm{e}-\mathrm{h}_{\mathrm{d}}}
\end{array}\right. \\
& \because k_{\text {up }}=k_{\text {up }}^{0} \exp \left(-\frac{\Delta E_{\mathrm{g}}}{k_{\mathrm{B}} T}\right)
\end{aligned}
$$




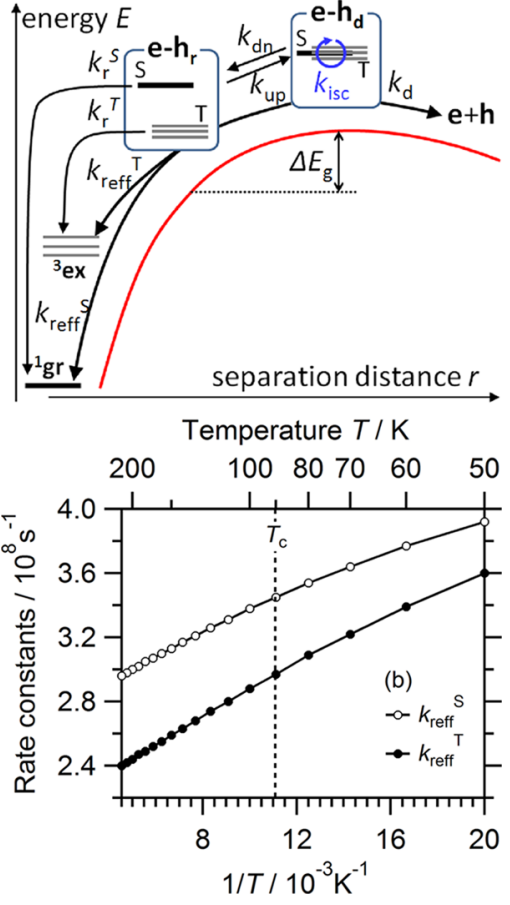

Figure 5. (a) Schematic diagram of the $\mathrm{e}-\mathrm{h}$ pair dynamics in carrier generation. The red curve is the potential energy due to Coulomb interaction and the external $E$ field. (b) Effective rate constants for the spin-selective geminate recombination obtained from the simulations of the temperature dependencies of $Q_{p}$ (Figure 3) and MPC (Figure 4).

$n_{\mathrm{e}-\mathrm{h}}$ and $k_{\mathrm{up}}{ }^{0}$ are, respectively, number density of the $\mathrm{e}-\mathrm{h}$ pair and the prefactor of the separation rate constant of $e-h_{r}$ following from the Arrhenius law. $k_{\mathrm{B}}$ is the Boltzmann constant. Because coherent $\mathrm{S}-\mathrm{T}$ mixing among the degenerate spin states of the $\mathrm{e}-\mathrm{h}$ pair is effective due to the hyperfine interaction $\left(A_{\mathrm{hf}}\right)$, isc does not occur in $\mathrm{e}-\mathrm{h}_{\mathrm{r}}$. When $B$ as much greater than $A_{\mathrm{hf}}$ is applied, the isc only between the $\mathrm{S}$ and $\mathrm{T}_{0}$ states of the $\mathrm{e}-\mathrm{h}_{\mathrm{d}}$ is allowed because of large energy shifts of the $\mathrm{T}_{ \pm}$states due to the Zeeman interaction. The observed MPC is caused by the combination of this magnetic fielddependent isc of $\mathrm{e}-\mathrm{h}_{\mathrm{d}}$ and the spin conservative recombination $\left(k_{\mathrm{r}}^{\mathrm{S}} \neq k_{\mathrm{r}}^{\mathrm{T}}\right)$ of $\mathrm{e}-\mathrm{h}_{\mathrm{r}}$, which recombines into ${ }^{1}$ gr or the ${ }^{3} \mathrm{ex}$. We take the magnetic field-dependent isc and the spin-selective recombination in the rate equations above (eq 1). By employing a steady-state approximation on $\mathrm{e}-\mathrm{h}_{\mathrm{r}}$ under an initial condition that ${ }^{1} \mathrm{ex}$ generates $\mathrm{e}-\mathrm{h}_{\mathrm{d}}$ quickly, the effective recombination rate constants from $\mathrm{e}-\mathrm{h}_{\mathrm{r}}\left(k_{\text {reff }}^{i}\right)$ are reduced as

$$
k_{\mathrm{reff}}^{i}=k_{\mathrm{dn}} \phi_{\mathrm{r}}^{i}=k_{\mathrm{dn}} \frac{k_{\mathrm{r}}^{i}}{k_{\mathrm{r}}^{i}+k_{\mathrm{up}}}
$$

$k_{\text {reff }}$ is proportional to the recombination yield of the $\mathrm{e}-\mathrm{h}_{\mathrm{r}}$ pair $\left(\phi_{\mathrm{r}}^{i}\right)$, which involves the thermally activated separation of $\mathrm{e}-\mathrm{h}_{\mathrm{d}}$ from $\mathrm{e}-\mathrm{h}_{\mathrm{r}}\left(k_{\mathrm{up}}\right)$. Eq 1 in two extreme cases can be consequently rewritten as

$$
\left\{\begin{aligned}
\frac{\mathrm{d} n_{\mathrm{e}-\mathrm{h}_{\mathrm{d}}}^{\mathrm{S}}=}{\mathrm{d} t} & -\left(3 k_{\mathrm{isc}}+k_{\text {reff }}^{\mathrm{S}}+k_{\mathrm{d}}\right) n_{\mathrm{e}-\mathrm{h}_{\mathrm{d}}}^{\mathrm{S}} \\
& +k_{\mathrm{isc}} \sum_{j=+, 0,-}^{\mathrm{T}_{\mathrm{e}-\mathrm{h}_{\mathrm{d}}}} \\
\frac{\mathrm{d} n_{\mathrm{e}-\mathrm{h}_{\mathrm{d}}}^{\mathrm{T}_{j}}}{\mathrm{~d} t}= & k_{\mathrm{isc}} n_{\mathrm{e}-\mathrm{h}_{\mathrm{d}}}^{\mathrm{S}}-\left(k_{\mathrm{isc}}+k_{\text {reff }}^{\mathrm{T}}+k_{\mathrm{d}}\right) n_{\mathrm{e}-\mathrm{h}_{\mathrm{d}}}^{\mathrm{T}_{j}}
\end{aligned}\right.
$$

at $B=0$ and

$$
\left\{\begin{aligned}
& \frac{\mathrm{d} n_{\mathrm{e}-\mathrm{h}_{\mathrm{d}}}^{\mathrm{S}}=}{\mathrm{d} t}-\left(k_{\mathrm{isc}}+k_{\mathrm{reff}}^{\mathrm{S}}+k_{\mathrm{d}}\right) n_{\mathrm{e}-\mathrm{h}_{\mathrm{d}}}^{\mathrm{S}} \\
&+k_{\mathrm{isc}} \sum_{j=+, 0,-} n_{\mathrm{e}-\mathrm{h}_{\mathrm{d}}}^{\mathrm{T}_{j}} \\
&\left\{\begin{array}{l}
\frac{\mathrm{d} n_{\mathrm{e}-\mathrm{h}}^{\mathrm{T}_{0}}}{\mathrm{~d} t}=k_{\text {isc }} n_{\mathrm{e}-\mathrm{h}_{\mathrm{d}}}^{\mathrm{S}}-\left(k_{\mathrm{isc}}+k_{\text {reff }}^{\mathrm{T}}+k_{\mathrm{d}}\right) n_{\mathrm{e}-\mathrm{h}_{\mathrm{d}}}^{\mathrm{T}_{0}} \\
\frac{\mathrm{d} n_{\mathrm{e}-h_{\mathrm{d}}}^{\mathrm{T}_{\mathrm{T}}}}{\mathrm{d} t}=-\left(k_{\mathrm{reff}}^{\mathrm{T}}+k_{\mathrm{d}}\right) n_{\mathrm{e}-\mathrm{h}_{\mathrm{d}}}^{\mathrm{T}_{ \pm}}
\end{array}\right.
\end{aligned}\right.
$$

at $B \gg A_{\mathrm{hf}}$. To interpret the time profile and temperature dependence of $Q$, we calculate the total density of free carriers $\left(n_{\mathrm{fc}}\right)$ that have escaped from $\mathrm{e}-\mathrm{h}_{\mathrm{d}}$

$$
\frac{\mathrm{d} n_{\mathrm{fc}}}{\mathrm{d} t}=k_{\mathrm{d}} \sum_{i=\mathrm{S}, \mathrm{T}_{+}, \mathrm{T}_{0}, \mathrm{~T}_{-}} n_{\mathrm{e}-\mathrm{h}_{\mathrm{d}}}^{i}
$$

The analytical expression of $n_{\mathrm{fc}}(t)$ under an initial condition of $n_{\mathrm{e}-\mathrm{hd}} \mathrm{S}=n_{0}$ and $n_{\mathrm{e}-\mathrm{hd}}{ }^{\mathrm{T}}=0$ is described in the literature. ${ }^{10} \mathrm{We}$ also calculate MPC due to the geminate pair using the formula

$$
\operatorname{MPC}_{\mathrm{g}}(B, t)=\frac{\int_{0}^{t} n_{\mathrm{fc}}(B, \tau) \mathrm{d} \tau-\int_{0}^{t} n_{\mathrm{fc}}(0, \tau) \mathrm{d} \tau}{\int_{0}^{t} n_{\mathrm{fc}}(0, \tau) \mathrm{d} \tau} \times 100
$$

to explain the temperature dependence of negative MPC of the geminate pair mechanism.

We carried out the simulations for both temperature dependence of the $Q_{p}$ below $T_{c}$ and the MPC of the geminate pair mechanism to obtain a unique set of rate constants. Because $k_{\text {isc }}\left(6.4 \times 10^{7} \mathrm{~s}^{-1}\right), k_{\mathrm{d}}\left(6.0 \times 10^{7} \mathrm{~s}^{-1}\right)$, and the ratio of $k_{\text {reff }}^{\mathrm{S}} / k_{\text {reff }}^{\mathrm{T}}(1.0: 0.8)$ at $298 \mathrm{~K}$ have been already estimated in the literature, ${ }^{10}$ there are three free parameters $k_{\mathrm{dn}}, k_{\mathrm{up}}^{0} / k_{\mathrm{r}}$, and $\Delta E_{\mathrm{g}}$ for the simulation. The black solid lines in Figures $3 \mathrm{~b}$ and $4 \mathrm{~b}$ are the best simulations that are respectively calculated with eqs 4 and 5. These simulations well reproduce the experimental results, and the estimated $k_{\text {reff }}$ constants are shown in Figure $5 \mathrm{~b}$. The observed decrease of $\mid \mathrm{MPCl}$ as lowering of $T$ is qualitatively understood by a trend of the $\mathrm{e}-\mathrm{h}$ pair equilibrium toward low-lying $\mathrm{e}-\mathrm{h}_{\mathrm{r}}$, which has no magnetic field-dependent isc because of the large $S-T$ energy gap. Also the phase and size of MPC are sensitive to the ratio of $k_{\text {reff }}^{S} / k_{\text {reff }}^{\mathrm{T}}$. The negative phase of MPC appears only under the condition of $k_{\text {reff }}^{\mathrm{S}}>k_{\text {reff }}^{\mathrm{T}}$. Hence, it can be said that the reduction of the difference between $k_{\text {reff }}^{S}$ and $k_{\text {reff }}^{\mathrm{T}}$ in the low $T$ regime of Figure $5 \mathrm{~b}$ is the cause for the decrease of $\mid \mathrm{MPCl}$ at low temperatures. Quantitatively lowering $T$ makes $k_{\text {up }}$ in eq 2 small, which equalizes $k_{\text {reff }} \mathrm{S}$ and $k_{\text {reff }}{ }^{\mathrm{T}}$. The $\Delta E_{\mathrm{g}}$ estimated from the simulations is $7 \mathrm{meV}$, which is smaller than that of poly(para-phenylene) film $(18-26 \mathrm{meV})$ obtained from temperature dependence of the magnetoluminescence effect 
at zero electric field ${ }^{23}$ because the potential barrier of the $e-h$ pair becomes small in presence of the $E$ field. This reasonable agreement of $\Delta E_{\mathrm{g}}$ in carrier generation of the organic solid thus speaks for the validity of the model on the basis of the equilibrium between the $e-h_{r}$ and $e-h_{d}$ pairs.

3.2. Carrier Transport. The temperature dependencies of $Q_{d}$ and MPC in the later times indicate that there is also the thermal activation process in carrier transport. Therefore, we discuss carrier transport in the self-assembly of HBC-C14 using the multiple trapping model (Figure 6a), which includes both

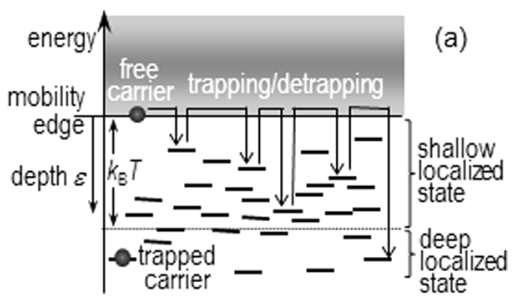

Temperature $T / \mathrm{K}$

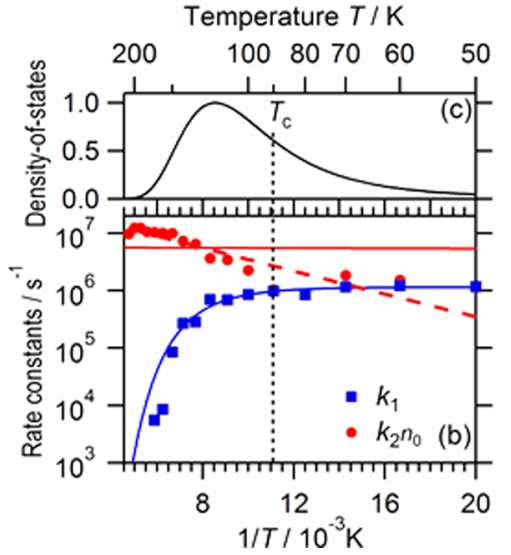

Figure 6. (a) Multiple trapping model for the carrier transport. The localized states in the band gap are divided into shallow and deep states according to $\varepsilon$, with the reference of $k_{\mathrm{B}} T$. (b) Temperature dependencies of the carrier elimination rate constants of $k_{1}$ (blue) and $k_{2} n_{0}$ (red) estimated by fitting the time profiles of $Q$ signal, depicted in Figure 3a. The solid curves are the simulations calculated using the multiple trapping model, with an energy distribution for density of localized states. The red broken line is calculated using an Arrhenius rate constant for $k_{2}$. (c) Gaussian distribution for density of the localized states optimized by fitting the temperature dependences in $b$.

the band conduction in ordered domains and hopping conduction in regions with no long-range order. ${ }^{24-26}$ Thus, there is trapping/detrapping dynamics and nongeminate recombination. The carriers above the mobility edge, called as the free carriers, transport along the $E$ field and they also may be captured into the localized states in the band gap during the transport. The carriers in the localized states, that is, the trapped carriers, do not move until detrapping to the mobility edge using the thermal energy of $k_{\mathrm{B}} T$, which can be the origin for the thermal activation process observed in the carrier transport. Because the carrier detrapping hardly takes place if the energy depth of the localized state $(\varepsilon)$ is deeper than that of $k_{\mathrm{B}} T$, carrier trappings into the deep-localized states eliminate the free carriers. On the other hand, the free carriers can form the nongeminate $\mathrm{e}-\mathrm{h}$ pairs by statistical encounters with other free carriers having opposite charge and then can disappear by nongeminate recombination. According to such carrier dynamics in the multiple trapping model, the rate equation for the free carrier density $\left(n_{\mathrm{fc}}\right)$ is written as

$$
\frac{\partial n_{\mathrm{fc}}}{\partial t}=-k_{1} n_{\mathrm{fc}}-k_{2} n_{\mathrm{fc}}^{2}
$$

The first- $\left(k_{1}\right)$ and second-order $\left(k_{2}\right)$ rate constants arise from carrier trapping into the deep-localized state and nongeminate recombination, respectively. To convert the solution of eq 6 to time evolution of the $Q_{d}$ signal, one must perform time integration of $n_{\mathrm{fc}}$ (see Section S3 in Supporting Information for details of the derivation). Because the total $Q$ signal consists of not only $Q_{d}$ but also $Q_{p}$, the final analytical form for the time profile is

$$
\begin{aligned}
& Q(t)=Q_{\mathrm{d}}+Q_{\mathrm{p}}=Q_{\mu}\left\{\frac{\ln \left[\mathrm{e}^{k_{1} t}-k_{2} n_{0} / k_{1}\left(1-\mathrm{e}^{k_{1} t}\right)\right]}{k_{2} n_{0}}\right. \\
& \left.-\frac{k_{1}}{k_{2} n_{0}} t\right\}+Q_{\mathrm{p}},\{t \mid t \geq 0\} \\
& \because Q_{\mu}=\frac{q \mu E S}{C}
\end{aligned}
$$

$n_{0}, q, \mu$, and $S$ represent initial densities of the free carriers, the elementary charge, drift mobility, and cross section of the carrier transport way, respectively. As shown in Figure 3a, eq 7a reproduced the observed time profiles of $Q$ very well. The $k_{1}$ and $k_{2} n_{0}$ constants optimized in the simulation at each temperature are plotted in Figure $6 \mathrm{~b}$. These rate constants are comparable to each other in the low $T$ regime. In the high $T$ regime, $k_{1}$ drops substantially but $k_{2} n_{0}$ increases with increasing $T$. Because carrier trapping is determined by a magnitude relation between $k_{\mathrm{B}} T$ and $\varepsilon$, the sudden drop of $k_{1}$ above the critical temperature suggests that $T_{c}$ reflects a depth for majority of the localized states.

In the multiple trapping model, as described in Section S3, the carrier trapping and recombination rates depend on not only $T$ but also the density of the localized states $g(\varepsilon)$. To calculate the temperature dependencies of $k_{1}$ and $k_{2}$, we adapted a Gaussian distribution, $g$

$$
g(\varepsilon)=\frac{2 N_{\mathrm{t}}}{\sqrt{\pi} \sigma_{\varepsilon}} \exp \left[-\left(\frac{\varepsilon-\varepsilon_{0}}{\sigma_{\varepsilon}}\right)^{2}\right]
$$

$N_{\mathrm{t}}$ is the total number of the localized states. As represented in Figure $6 \mathrm{~b}$, the calculation using eq $S 9$ reproduced the temperature dependence of $k_{1}$ but did not duplicate well that of $k_{2} n_{0}$. The fit of the calculation of $k_{2} n_{0}$ with the observed one is improved by employing an Arrhenius type of recombination rate constant, $\beta n_{0}=\beta_{0} \phi_{\text {adj }} \exp \left(-\Delta E_{\mathrm{ngr}} / k_{\mathrm{B}} T\right)$ into eq S9b. Here, $\phi_{\text {adj }}$ is the temperature dependence of the dimensionless carrier generation efficiency estimated from the prompt MPC in Figure $4 \mathrm{~b}$ for taking into account the temperature effect on $n_{0}$. The shape of $g$ and the parameters determined by the simulations are, respectively, shown in Figure $6 \mathrm{c}$ and Table 1.

Depth at the distribution peak $\left(\varepsilon_{0}\right)$ of the HBC-C14 assembly is estimated to be $10.1 \mathrm{meV}$, which is slightly larger than that of the thermal energy at $T_{c}$ of $90 \mathrm{~K}$ and is shallower

Table 1. Parameters of Gaussian Distribution for the Density of Localized States and $k_{2} n_{0}$ Obtained from the Simulation of the Temperature Dependence of $k_{1}$ and $k_{2} n_{0}$

$\begin{array}{cccc}\varepsilon_{0} / \mathrm{meV} & \sigma_{\varepsilon} / \mathrm{meV} & \beta_{0} / 10^{8} \mathrm{~s}^{-1} & \Delta E_{\mathrm{ngr}} / \mathrm{meV} \\ 10.1 & 3.3 & 2.1 & 18.9\end{array}$


than that for randomly oriented films consisting of polymers $(350-400 \mathrm{meV})^{27}$ or small molecules $(190-400 \mathrm{meV})^{28,29}$ and for blend films of poly(3-hexylthiophene-2,5-diyl) (P3HT) and $[6,6]$-phenyl- $\mathrm{C}_{61}$-butyric acid methyl ester $\left(\mathrm{PC}_{61} \mathrm{BM}\right)(87$ $\mathrm{meV}){ }^{30}$ The estimated distribution width $\left(\sigma_{\varepsilon}\right)$ of $3.3 \mathrm{meV}$ for the HBC-C14 assembly is narrower than that of the polymers $(200-500 \mathrm{meV})^{27}$ and small molecules (100-150 $\mathrm{meV})^{28,29}$ and the blend film of $\mathrm{P} 3 \mathrm{HT} / \mathrm{PC}_{61} \mathrm{BM}(29 \mathrm{meV}){ }^{30}$ The organized structure of $\mathrm{HBC}-\mathrm{C} 14$ in the one-dimensional assembly is the main reason for such shallow depth and narrow distribution. Because in the high $T$ regime most of the delocalized states behave as shallow traps, $n_{\mathrm{fc}}$ efficiently increases with increasing $T$. On the other hand, the thermal activation of $\Delta E_{\mathrm{ngr}}=18.9 \mathrm{meV}$ obviously indicates that the increase of the nongeminate recombination rate as a bimolecular reaction in high temperatures is due to the increase of not only $n_{\mathrm{fc}}$ but also $k_{2} . \Delta E_{\mathrm{ngr}}$ is comparable to the apparent activation energy $\Delta E^{\mathrm{HT}}$ in the high $T$ regime. The presence of activation energy in the nongeminate recombination rate constant is interpreted in terms of the Langevin bimolecular recombination, in which the rate factor is a function of the drift mobility activated by thermal energy. ${ }^{31}$

\section{CONCLUSIONS}

Using the time-resolved MPC technique, we examined the temperature dependence of carrier generation and transport in the low-dimensional molecular assembly of HBC-C14. The Arrhenius analysis of $Q$ and MPC turned out to show a small activation energy for the carrier generation and a large one for carrier transport. The carrier generation is basically interpreted in terms of competing kinetics between the recombination and dissociation of the geminate $\mathrm{e}-\mathrm{h}$ pair on the singlet and triplet Coulomb potentials distorted by the applied $E$ field. An equilibrium dynamics between the short- and long-distance e$\mathrm{h}$ pairs has been clarified. For carrier transport, on the other hand, the observed thermal activation of the transient $Q$ signal was understood by the multiple trapping model taking account of both the unimolecular decay of carriers due to the trapping and Langevin bimolecular recombination. It has been elucidated that the HBC-C14 assembly has a narrow energy distribution of the shallow localized states, which is an important advantage of the ordered molecular system.

\section{EXPERIMENTAL SECTION}

HBC-C14 was synthesized as reported previously. ${ }^{32,33}$ The film of the fiber assembly of the HBC-C14 columns was prepared on quartz glass plates coated with comb-shaped indium tin oxide electrodes, with a gap of $5.0 \mu \mathrm{m}$ by a drop cast method, using dichloromethane solution ${ }^{34}$ (see Figure S3). The film sample on the glass plate was encapsulated with a cover glass plate to avoid exposure to atmospheric oxygen. Details of the setup for time-resolved MPC measurement in Q-mode are described in the literature. ${ }^{35}$ The transient $Q$ signal induced by irradiation using nanosecond lasers was measured using an $R C$ circuit comprising the sample device, with a capacitance $(C)$ of ca. $150 \mathrm{pF}$, a resistance $(R)$ of $1.0 \mathrm{M} \Omega$, and a variable DC power supply. The voltage generated between both ends of the $R$, which is induced by the drift of photocarriers and corresponds to $Q / C$, was monitored as a function of the delay time after laser flash. A static $B$ field was applied to a film fixed in the center of an electromagnet to observe the MPC effect. Temperature of the sample was controlled by a He flow cryostat equipped with quartz windows for light irradiation (Optistat CF, Oxford Instruments).

\section{ASSOCIATED CONTENT}

\section{Supporting Information}

The Supporting Information is available free of charge on the ACS Publications website at DOI: 10.1021/acsomega.7b00474.

Magnetic field dependence of magnetophotoconductance effect (S1); electric field dependence of the dissociation efficiency from a geminate electron-hole pair (S2); carrier transport with trapping and nongeminate recombination (S3); rate constants $k_{1}$ and $k_{2}$ in Gaussian density of the localized states (S4); fiber assembly of hexabenzocoronene attached with six tetradecyl chains (S5) (PDF)

\section{AUTHOR INFORMATION}

\section{Corresponding Author}

*E-mail: ikoma@chem.sc.niigata-u.ac.jp. Phone/Fax: +81-25262-7390.

ORCID $\odot$

Tadaaki Ikoma: 0000-0003-2003-6839

Yohei Yamamoto: 0000-0002-2166-3730

Takanori Fukushima: 0000-0001-5586-9238

\section{Notes}

The authors declare no competing financial interest.

\section{ACKNOWLEDGMENTS}

Y.W. and T.I. thank Prof. Dr. K. Shinbo (Niigata Univ.) for helping in the measurement of the optical absorption spectrum. This work was performed under the Cooperative Research Program of "Network Joint Research Center for Materials and Devices: Dynamic Alliance for Open Innovation Bridging Human, Environment and Materials" and was financially supported by Grants-in-Aid for JSPS Fellows (No. 23-7285), JSPS KAKENHI Grants (Nos. 26410088, 16KT0049, and 17K14477).

\section{REFERENCES}

(1) Clarke, T. M.; Durrant, J. R. Charge Photogeneration in Organic Solar Cells. Chem. Rev. 2010, 110, 6736-6767.

(2) Pope, M.; Swenberg, C. E. Electronic Processes in Organic Crystals and Polymers, 2nd ed.; Oxford University Press: New York, 1999.

(3) Hayashi, H. Introduction to Dynamic Spin Chemistry: Magnetic Field Effects on Chemical and Biochemical Reactions; World Scientific Pub. Co. Inc.: Singapore, 2004.

(4) Vardeny, Z. V., Organic Spintronics, 1st ed.; CRC Press: 2010; p 335.

(5) Shiraishi, M.; Ikoma, T. Molecular Spintronics. Phys. E 2011, 43, 1295-1317.

(6) Watson, M. D.; Fechtenkotter, A.; Müllen, K. Big Is Beautiful "Aromaticity" Revisited from the Viewpoint of Macromolecular and Supramolecular Benzene Chemistry. Chem. Rev. 2001, 101, 12671300.

(7) Wu, J.; Pisula, W.; Müllen, K. Graphenes as Potential Material for Electronics. Chem. Rev. 2007, 107, 718-747.

(8) van de Craats, A. M.; Warman, J. M.; Müllen, K.; Geerts, Y.; Brand, J. D. Rapid Charge Transport along Self-Assembling Graphitic Nanowires. Adv. Mater. 1998, 10, 36-38.

(9) van de Craats, A. M.; Stutzmann, N.; Bunk, O.; Nielsen, M. M.; Watson, M.; Müllen, K.; Chanzy, H. D.; Sirringhaus, H.; Friend, R. H. Meso-Epitaxial Solution-Growth of Self-Organizing Discotic LiquidCrystalline Semiconductors. Adv. Mater. 2003, 15, 495-499. 
(10) Wakikawa, Y.; Ikoma, T.; Yamamoto, Y.; Fukushima, T.; Aida, T.; Akiyama, K. Effect of Acceptor Lamination on Photocarrier Dynamics in Hole Transporting Hexabenzocoronene Nanotubular Self-assembly. J. Phys. Chem. C 2013, 117, 15295-15305.

(11) Piris, J.; Debije, M. G.; Watson, M. D.; Müllen, K.; Warman, J. M. The Mobility and Decay Kinetics of Charge Carriers in Discotic Hexabenzocoronenes. Adv. Funct. Mater. 2004, 14, 1047-1052.

(12) Schmidt-Mende, L.; Fechtenkotter, A.; Müllen, K.; Moons, E.; Friend, R. H.; MacKenzie, J. D. Self-Organized Discotic Liquid Crystals for High-Efficiency Organic Photovoltaics. Science 2001, 293, $1119-1122$.

(13) Piris, J.; Debije, M. G.; Stutzmann, N.; van de Craats, A. M.; Watson, M. D.; Müllen, K.; Warman, J. M. Anisotropy in the Mobility and Photogeneration of Charge Carriers in Thin Films of Discotic Hexabenzocoronenes, Columnarly Self-Assembled on Friction-Deposited Poly(tetrafluoroethylene). Adv. Mater. 2003, 15, 1736-1740.

(14) Hill, J. P.; Jin, W. S.; Kosaka, A.; Fukushima, T.; Ichihara, H.; Shimomura, T.; Ito, K.; Hashizume, T.; Ishii, N.; Aida, T. SelfAssembled Hexa-peri-Hexabenzocoronene Graphitic Nanotube. Science 2004, 304, 1481-1483.

(15) Steiner, U. E.; Ulrich, T. Magnetic-field Effects in Chemicalkinetics and Related Phenomena. Chem. Rev. 1989, 89, 51-147.

(16) Onsager, L. Initial Recombination of Ions. Phys. Rev. 1938, 54, 554-557.

(17) Melz, P. J. Photogeneration in Trinitrofluorenone-Poly $(N$ Vinylcarbazole). J. Chem. Phys. 1972, 57, 1694-1699.

(18) Mozumder, A. Effect of an External Electric Field on the Yield of Free Ions. I General Results from the Onsager Theory. J. Chem. Phys. 1974, 60, 4300-4304.

(19) Braun, C. L. Electric-Field Assisted Dissociation of Chargetransfer States as a Mechanism of Photocarrier Production. J. Chem. Phys. 1984, 80, 4157-4161.

(20) Hong, K. M.; Noolandi, J. Solution of Time-Dependent Onsager Problem. J. Chem. Phys. 1978, 69, 5026-5039.

(21) Wojcik, M.; Tachiya, M. Geminate Charge Recombination with Distance-Dependent Intrinsic Reaction Rate: Escape Probability and its Electric Field Effect. Radiat. Phys. Chem. 2005, 74, 132-138.

(22) Barth, S.; Hertel, D.; Tak, Y.-H.; Bässler, H.; Hörhold, H. H. Geminate Pair Dissociation in Random Organic Systems. Chem. Phys. Lett. 1997, 274, 165-170.

(23) Frankevich, E. L.; Zoriniants, G. E.; Chaban, A. N.; Triebel, M. M.; Blumstengel, S.; Kobryanskii, V. M. Magnetic Field Effects on Photoluminescence in PPP. Investigation of the Influence of Chain Length and Degree of Order. Chem. Phys. Lett. 1996, 261, 545-550.

(24) Arkhipov, V. I.; Rudenko, A. I. Drift and Diffusion in Materials with Traps. Philos. Mag. B 1982, 45, 189-207.

(25) Arkhipov, V. I.; Kolesnikov, V. A.; Rudenko, A. I. Dispersive Transport of Charge Carriers in Polycrystalline Pentacene Layers. J. Phys. D: Appl. Phys. 1984, 17, 1241.

(26) Ansari-Rad, M.; Anta, J. A.; Bisquert, J. Interpretation of Diffusion and Recombination in Nanostructured and EnergyDisordered Materials by Stochastic Quasiequilibrium Simulation. J. Phys. Chem. C 2013, 117, 16275-16289.

(27) von Malm, N.; Schmechel, R.; von Seggern, H. Invited Paper: Electronic Traps in Polymeric Semiconductors. SID Int. Symp. Dig. Tech. Pap. 2003, 34, 1072-1075.

(28) Schmechel, R.; von Seggern, H. Electronic Traps in Organic Transport Layers. Phys. Status Solidi A 2004, 201, 1215-1235.

(29) Steiger, J.; Schmechel, R; von Seggern, H. Energetic Trap Distributions in Organic Semiconductors. Synth. Met. 2002, 129, 1-7.

(30) Tajima, H.; Suzuki, T.; Kimata, M. Direct Determination of Trap Density Function Based on the Photoinduced Charge Carrier Extraction Technique. Org. Electron. 2012, 13, 2272-2280.

(31) van der Holst, J. J. M.; van Oost, F. W. A.; Coehoorn, R.; Bobbert, P. A. Electron-hole Recombination in Disordered Organic Semiconductors: Validity of the Langevin formula. Phys. Rev. B 2009, 80, No. 235202.

(32) Motoyanagi, J.; Yamamoto, Y.; Saeki, A.; Alam, M. A.; Kimoto, A.; Kosaka, A.; Fukushima, T.; Seki, S.; Tagawa, S.; Aida, T. Unusual
Side-Chain Effects on Charge-Carrier Lifetime in Discotic Liquid Crystals. Chem. - Asian J. 2009, 4, 876-880.

(33) van de Craats, A. M.; Warman, J. M.; Fechtenkotter, A.; Brand, J. D.; Harbison, M. A.; Müllen, K. Record Charge Carrier Mobility in a Room-temperature Discotic Liquid-crystalline Derivative of Hexabenzocoronene. Adv. Mater. 1999, 11, 1469-1472.

(34) Fischbach, I.; Pakula, T.; Minkin, P.; Fechtenkötter, A.; Müllen, K.; Spiess, H. W.; Saalwächter, K. Structure and Dynamics in Columnar Discotic Materials: A Combined X-ray and Solid-State NMR Study of Hexabenzocoronene Derivatives. J. Phys. Chem. B 2002, 106, 6408-6418.

(35) Ikoma, T.; Ogiwara, T.; Takahashi, Y.; Akiyama, K.; TeroKubota, S.; Takahashi, Y.; Suzuki, T.; Wakikawa, Y. Giant Magnetoresistance due to Electron-hole Pair Mechanism in Poly $(N$-vinylcarbazole). Synth. Met. 2010, 160, 285-290. 